\title{
Incidence and Pathology of Paratyphoid Infection in Poultry
}

\author{
Anushri Tiwari, Madhu Swamy, Yamini Verma, Amita Dubey
}

10.18805/IJAR.B-4460

\begin{abstract}
Background: Paratyphoid infection of poultry is caused by non-host adapted motile salmonellae and are responsible for numerous cases of food borne illness worldwide. The present study was carried out from July 2019 to February 2020 in Jabalpur to know the occurrence and pathology of paratyphoid bacteria in poultry.

Methods: Whole blood agglutination test was performed to know the prevalence of salmonellosis in and areas surrounding Jabalpur region and pooled fecal samples were collected from poultry farms to perform microbe culture and biochemical characterization. Serotyping of Salmonella isolates was done using polyvalent antisera. Necropsy examination was conducted to observe gross and histopathological lesions.

Conclusion: Rapid whole blood agglutination test determined the percent prevalence of Salmonella as $28.0 \%$ from 25 private poultry outlets. The percent prevalence of salmonellosis by collecting pooled fecal samples from 15 broiler and 11 layer farms was recorded as $20.0 \%$ and $45.4 \%$ respectively. Salmonellosis was recorded in $1.58 \%$ of total necropsy cases of birds examined for gross and histopathological studies. Polyvalent antisera diagnosed $27.27 \%$ motile paratyphoid salmonellae, out of which $18.18 \%$ tested positive for Salmonella Enteritidis while $9.09 \%$ tested positive for Salmonella Typhimurium. Birds with paratyphoid infection showed hepatomegaly, discoloration, hemorrhagic and necrotic foci in liver and various grades of hemorrhagic to catarrhal enteritis were recorded.

Key words: Paratyphoid, Poultry, Salmonella, S. Enteritidis.
\end{abstract}

\section{INTRODUCTION}

Indian poultry industry is one of the world's largest and fastest growing industry ranking third in egg production and the fourth largest chicken meat producer in the world (Prabakaran, 2014). Modernization of livestock farms and globalization of bird breeding trade also helps in transboundary spreading of food-borne bacteria such as Salmonella (Chakraborty et al., 2020). Although, the poultry sector contributes its great share to the country's economy, the production is hampered by several disease conditions. Salmonella infection is one of the most important bacterial diseases in poultry causing heavy economic loss through mortality and reduced production (Haider et al., 2004). NTS (Non-Typhoidal Salmonella) is one of the most prevalent foodborne infections around the world, causing diarrhea, fever, vomiting and sometimes even death. WHO has estimated that the NTS is responsible for an average number of 78.7 million foodborne diseases with more than 59000 deaths annually (Havelaar et al., 2015). There is relatively fewer number of reports of salmonellosis from India despite its high prevalence, which can be attributed to limited diagnostic facilities under field conditions and underreporting (Rajagopal and Mini, 2013). Paratyphoid infections of fowl may also reach the human population via contamination and mishandling of poultry products causing serious health issues.

Keeping the following facts in mind, the present study was conducted to determine the occurrence and pathology of paratyphoid infection in poultry in Jabalpur region of Madhya Pradesh.

\section{MATERIALS AND METHODS}

The present research work was conducted for a period of
Department of Veterinary Pathology, College of Veterinary Science and Animal Husbandry, NDVSU, Jabalpur-482 001, Madhya Pradesh, India.

Corresponding Author: Anushri Tiwari, Department of Veterinary Pathology, College of Veterinary Science and Animal Husbandry, Nanaji Deshmukh Veterinary Science University, Jabalpur-482 001, Madhya Pradesh, India. Email: t.anushri1226@gmail.com

How to cite this article: Tiwari, A., Swamy, M., Verma, Y. and Dubey, A. (2021). Incidence and Pathology of Paratyphoid Infection in Poultry. Indian Journal of Animal Research. DOI: 10.18805/IJAR.B-4460.

Submitted: 22-03-2021 Accepted: 19-07-2021 Online: 30-07-2021

eight months from July 2019 to February 2020, at the Department of Veterinary Pathology, College of Veterinary Science and Animal Husbandry, NDVSU, Jabalpur (M.P.) to determine the occurrence and pathology of paratyphoid Salmonella infection in poultry.

\section{Seroprevalence of Salmonella using whole blood agglutination test}

The seroprevalence of salmonellosis was recorded in 25 retail poultry outlets using Salmonella coloured antigen. Blood was collected from birds at the time of slaughter. A drop of coloured antigen was taken and then a drop of blood was added to the antigen and mixed and rocked gently for one minute and observed for agglutination reaction.

\section{Isolation of organism from poultry farms}

A total of 26 farms including 15 broiler farms and 11 layer farms were visited covering approximately $100 \mathrm{~km}$ radius area in and around Jabalpur region. Proper data collection 
was performed through interaction with farm manager and several aspects regarding health status of birds, biosecurity measures and other prevailing management practices.

Isolation of Salmonella was performed according to the protocol followed by ISO (2017). Pooled faecal samples $(100 \mathrm{~g})$ were collected from different sheds of individual poultry farms in sterile sample bottles containing $100 \mathrm{ml}$ of buffered peptone water (BPW) and then transported in iceboxes at $4^{\circ} \mathrm{C}$ and then the samples were incubated overnight at $37^{\circ} \mathrm{C}$. Liver and intestinal swab samples were also collected at the time of necropsy. Both samples were then inoculated into Tetrathionate broth and Rappaport Vassiliadis Medium and incubated at $37^{\circ} \mathrm{C}$ and $42^{\circ} \mathrm{C}$ respectively, for 18-24 hours for selective enrichment of the organism. Organisms from the broth medium were then streaked on to selective media XLD and BGA and incubated at $37^{\circ} \mathrm{C}$ for overnight for isolation of organism.

\section{Identification of organism}

Identification of organism was done based on study of characteristics of colony morphology and further Gram's staining was performed using pure colonies. Biochemical characterization of bacterial isolates was done using IMViC test and Triple Sugar Iron (TSI) agar test and metabolic changes that were interpreted visually.

\section{Identification of motile Salmonella organisms by use of polyvalent antisera}

A presumptive identification of Salmonella was done by using commercially available antisera (SSI Diagnostica, Denmark) specific for S. Enteritidis and S. Typhimurium. A small drop of antiserum $(20 \mu \mathrm{l})$ was added on a glass slide. A small amount of positive Salmonella culture was taken and then mixed with the antiserum. The slide was then tilted for 5-10 seconds. A positive reaction was seen as visible agglutination, whereas a negative reaction was observed as homogeneous milky turbidity.

\section{Pathological examination}

Examination of carcasses of birds (approximately $>200$ ) received at Department of Veterinary Pathology, C.O.V.Sc. and A.H., NDVSU, Jabalpur, was conducted for the presence of gross gastrointestinal lesions associated with paratyphoid infection during post mortem. For histopathology, the formalin fixed tissues of liver spleen, intestine and ovary from birds found positive for Salmonella infection were further processed, trimmed, sectioned and stained using routine Haematoxylin and Eosin Staining following standard procedure and presence of the bacteria in intestinal sections was determined by MacCallum Goodpasture staining as described by Gridley (1960).

\section{Statistical analysis}

Data gathered during the study was utilized for the analysis of the presence of disease within layers and broilers of various farms using Chi-square test as described by Snedecor and Cochran (1994).

\section{RESULT AND DISCUSSION}

The present work was conducted to determine the occurrence and pathology of paratyphoid infection in poultry farms and from the necropsy cases.

Seroprevalence of Salmonella using whole blood agglutination test

In the whole blood agglutination test, the positive agglutinates were of variable degrees in the outlets. On the basis of the whole blood agglutination test the seroprevalence of Salmonella in Jabalpur was determined as $28.0 \%$. In India, there is a practice of procuring the chicken meat from small retail outlets with a general observation of severe lack of hygiene and cleanliness.

Similar grading was used by Habib-ur-Rehman et al. (2003) and Rahman et al. (2011). However, this rapid detection test alone cannot give the accurate prevalence of salmonellosis infection. Due to the high sensitivity and low specificity of the whole blood agglutination test, it is widely considered to be of importance only as a screening test (Gast, 1997).

\section{Prevalence of Salmonella at poultry farms}

All the broiler farms followed deep litter system of housing for birds while cage system was followed in the layer farms. Based on the cultural and biochemical characteristics, salmonellosis was recorded in $1.58 \%$ (30/189) of necropsy cases (isolates named P1, P2 and P3). The Salmonella infection was confirmed in $20.0 \%(3 / 15)$ in broiler farms (B1, B2 and B3) and $45.45 \%$ $(5 / 11)$ in layer farms (L1, L2, L3, L4 and L5).

\section{Colony morphology}

Isolates of Salmonella sp. were observed as 2-3 mm pinkish red colonies with black centre on XLD Agar along with change of colour of media showing reddish appearance as observed by Ranjbar et al. (2020). In BGA, the Salmonella isolates appeared as pinkish white colonies with change of the colour of agar medium from green to pink similar to observations of Sannat et al. (2017).

\section{Gram staining of pure colonies}

All the suspected isolates showed the presence of pink coloured, small rod-shaped bacteria present alone or in groups from the pure colonies after observing under oil immersion in microscope. Salmonella was confirmed by presence of Gram-negative pink coloured rods after analysing Gram-stained smears (Tille, 2017).

\section{Biochemical characterization of Salmonella isolates}

The TSI test performed using the Salmonella isolates obtained showed a yellow coloured butt with a pink slant alkaline reaction and yellow butt showing acid production (Table 1) which indicates that the organism is a dextrose fermenter but unable to ferment lactose and sucrose. Black coloured growth was present indicating $\mathrm{H}_{2} \mathrm{~S}$ production. Gas production was denoted by presence of bubbles in the butt region. This has been described in concurrent studies done by Mansour et al. (2013) and Saravanan et al. (2015). 
Indole, Methyl red, Voges-Prausker and Citrate utilization (IMViC) Test

The biochemical characteristics of IMViC test of the isolates were in accordance with reactions mentioned for Salmonella $(-+-+)$ (OIE, 2012) as mentioned in Table 2.

All the isolates were found to be negative for indole test. Formation of red colour was noted when methyl red added to the MR-VP broth which showed positive methyl red test as observed by Faisal et al. (2017). The presented negative Voges-Prausker (VP) reaction. Similar result was found by Sharma and Das (2016). Colour change from green to blue in case of positive citrate utilization test was observed except for one isolate which was found to be indole negative. Negative result for citrate utilization by S. Gallinarum was also noted by Mir et al. (2015).

\section{Motility test}

Motile Salmonella were identified by the development of dark pink growth and movement of bacteria from the inoculated well no. 1 to well no. 2 in the kit unlike the nonmotile Salmonella (Table 2). A positive result of motility was indicated by the spread of the stab line in the solid media as stated by Aktar et al. (2016). Out of the 11 suspected isolates, $27.27 \%$ were identified as motile Salmonella.

\section{Serogrouping of Salmonella isolates using polyvalent} antisera

Salmonella somatic $O$ poly antisera specific for motile organisms S. Enteritidis and S. Typhimurium were used to

Table 1: Biochemical features of Salmonella isolates using TSI slant test.

\begin{tabular}{lccccc}
\hline Isolates & & Butt & Slant & $\begin{array}{c}\text { Gas } \\
\text { production }\end{array}$ & $\begin{array}{c}\mathrm{H}_{2} \mathrm{~S} \\
\text { production }\end{array}$ \\
\hline PM cases & P1 & Y & P & - & + \\
& P2 & B & B & + & + \\
Broiler farms & P3 & Y & P & + & + \\
& B1 & Y & P & + & + \\
& B2 & Y & P & - & + \\
Layer farms & B3 & B & P & + & + \\
& L1 & Y & P & + & + \\
& L2 & B & B & - & + \\
& L3 & Y & P & + & + \\
& L4 & Y & P & + & + \\
& L5 & B & P & - & + \\
\hline
\end{tabular}

$\mathrm{Y}=$ yellow, $\mathrm{B}=$ black, $\mathrm{P}=$ Pink, $+=$ present and $-=$ absent. differentiate the cultures having motile Salmonella from the non-motile organisms. Among the 11 isolates obtained, $18.18 \%$ tested positive for Salmonella Enteritidis antisera. Al Mamun et al. (2017) reported prevalence of Paratyphoid Salmonella as $27.77 \%$ and Long et al. (2017) reported it as $4.54 \%$.

In our study, 9.09\% tested positive for Salmonella Typhimurium antisera. Earlier, the seroprevalence of Salmonella Typhimurium reported by Srinivasan et al. (2014) and Muna et al. (2016) was $2.35 \%$ and $1.9 \%$ respectively, whereas Mir et al. (2015) and Khasa et al. (2018) reported the prevalence of Salmonella Typhimurium as $15.62 \%$ and $81.25 \%$ respectively. However, a similar prevalence of $9.09 \%$ of Salmonella Typhimurium was recorded by Samanta et al. (2014) in West Bengal.

Percentage positive samples of Paratyphoid Salmonella from broiler farms, layer farms and post-mortem cases with gastrointestinal lesions was recorded as $6.66 \%, 9.09 \%$ and $0.52 \%$ respectively. In accordance with our observation, it has been reported earlier also that with increased flock size of birds, Salmonella infection rate also increases (Samanta et al., 2014).

\section{Pathological examination}

\section{- Gross lesions of in birds affected with paratyphoid infection}

Out of 189 necropsy cases, 15 broiler birds were found positive for paratyphoid Salmonella infection. The birds were received with the history of anorexia, restlessness, dullness, depression and diarrhoea, however, no characteristic clinical signs were noted in the diseased birds from the farms.

Majority of gross lesions in birds were comprised of congestion of liver (43.33\%), congestion of spleen (36.66\%) and hemorrhagic enteritis $(40.0 \%)$ due to bacterial spread. Liver included lesions of hepatomegaly, congestion and hemorrhagic and necrotic foci in liver. Splenomegaly along with congestion and mottling of spleen was observed. The caeca were inflamed and swollen. Severe haemorrhagic gastroenteritis and haemorrhagic typhlitis along with haemorrhagic caecal tonsils was observed.

There is immense scope for contamination in the birds slaughtered in unorganized retail shops due to poor hygiene (Badhe et al., 2013). The two most consistently observed features of paratyphoid infections in mature poultry are intestinal colonization and systemic dissemination to internal organs (Gast, 2013). Crhanova et al. (2011) observed that

Table 2: Biochemical characterization of Salmonella isolates.

\begin{tabular}{|c|c|c|c|c|c|c|c|c|c|c|c|}
\hline \multirow{3}{*}{$\begin{array}{l}\text { Biochemical } \\
\text { Test }\end{array}$} & \multicolumn{11}{|c|}{ Isolates of Salmonella from different sources } \\
\hline & \multicolumn{3}{|c|}{ Post-mortem cases } & \multicolumn{3}{|c|}{ Broiler farms } & \multicolumn{5}{|c|}{ Layer farms } \\
\hline & $\mathrm{P} 1$ & P2 & P3 & B1 & B2 & B3 & L1 & L2 & L3 & L4 & L5 \\
\hline Indole & - & - & - & - & - & - & - & - & - & - & - \\
\hline Methyl red & + & + & + & + & + & + & + & + & + & + & + \\
\hline Voges-prausker & - & - & - & - & - & - & - & - & - & - & - \\
\hline Citrate & + & + & + & + & + & + & + & + & + & - & + \\
\hline Motility & + & - & - & + & - & - & + & - & - & - & - \\
\hline
\end{tabular}


infection with $\mathrm{S}$. Enteritidis resulted in significant inflammation which also required a strengthening of epithelial cell resistance and causes dissemination to internal organs.

\section{- Histopathological lesions in birds with Paratyphoid infection}

\section{Liver}

Microscopic lesions in liver included haemorrhagic foci, hemosiderosis, congestion, dilatation of sinusoids (Fig 1), cellular infiltration and vacuolar degeneration. Kupffer cell hypertrophy was also noted. Coagulative necrosis was also observed causing loss of organ structure in the liver sections (Fig 2). Liver was noted with maximum histopathological alterations in our study.

Presence of multifocal necrosis is an irreversible pathologic alteration (Garcia et al. 2013). Hepatic degeneration was also noted by Ogunleye and Carlson (2012). Focal degenerative and infiltrative lesions were observed by Hossain et al. (2006) and Dutta et al. (2013).

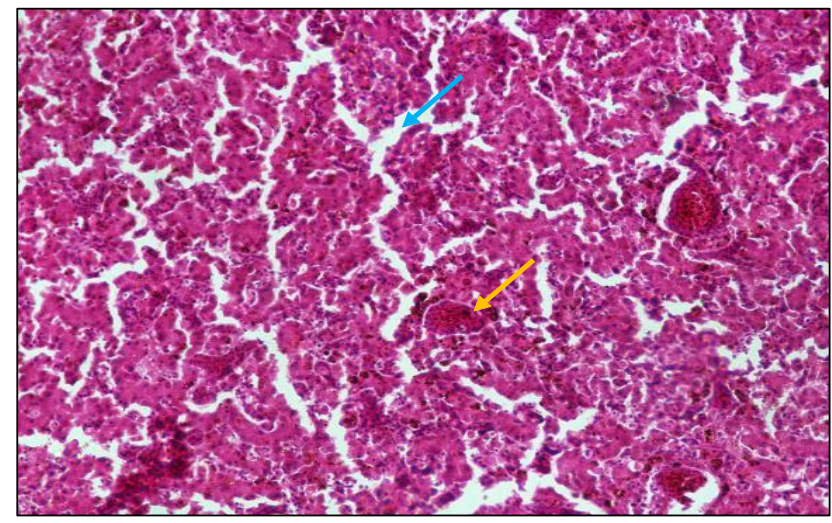

Fig 1: Microscopic section of liver from bird with paratyphoid infection showing congested central vein (yellow arrow) along with dilated and tortuous sinusoids (blue arrow). H \& E x 200.

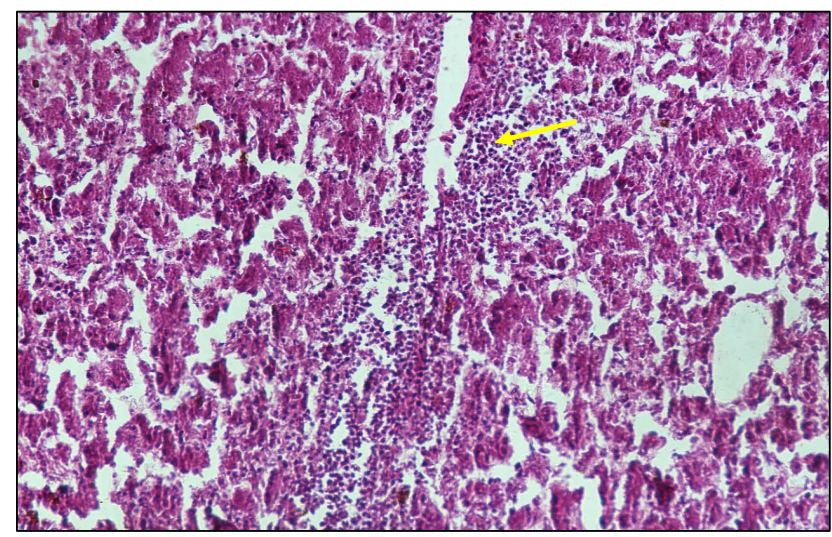

Fig 2: Microscopic section of liver from bird with paratyphoid infection showing large area of polymorph infiltration (yellow arrow) and necrosis. H \& E x 200.

\section{Spleen}

Spleen showed lymphocytic follicle depletion (Fig 3), fibrinoid necrosis and micro haemorrhages. Similar findings reported by Islam et al. (2006) and Kumari et al. (2013). Presence of severe congestion, haemorrhage and hemosiderosis on the spleen noted by Dutta et al. (2013) and Muna et al. (2016). Deshmukh et al. (2007) reported microscopic changes of congestion, depletions of lymphocytes from white pulp areas with RE cell hyperplasia and scattered infiltration of granulocytes in red pulp of spleen in Japanese quails.

\section{Intestine}

Intestine showed haemorrhages, goblet cell hyperplasia and desquamation of epithelium (Fig 4). Intense cellular infiltration in the caeca and intestine was observed. The findings were comparable to the microscopic lesions observed by Muna et al. (2016). Haemorrhages with infiltration of mononuclear cells in the intestinal submucosa were also observed in our study similar to the findings of Dutta et al. (2015).

Burkholder et al. (2008) discussed that acute stressor can lead to significant changes in the normal intestinal

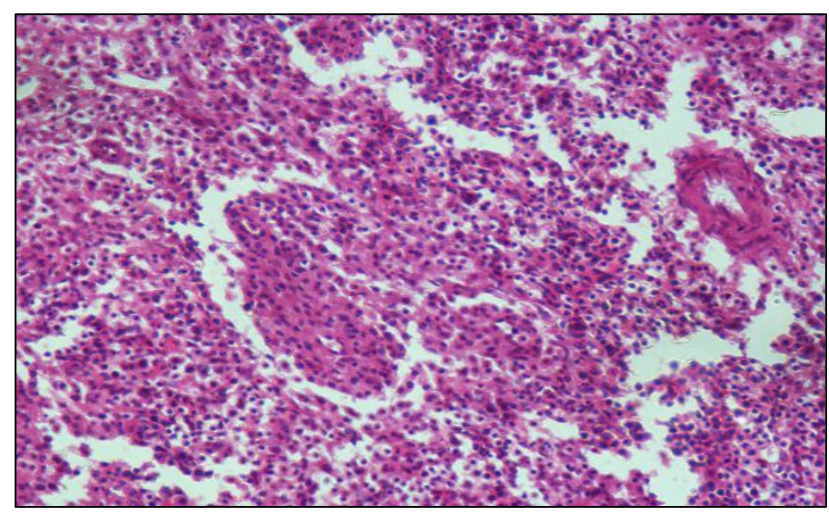

Fig 3: Microscopic section of spleen from bird with paratyphoid infection showing lymphocytic follicle depletion. H \& E x 400 .

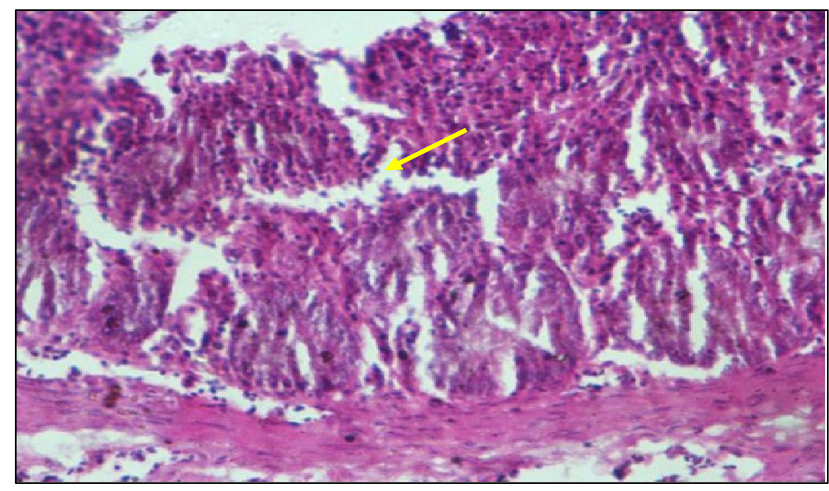

Fig 4: Microscopic section of intestine from bird with paratyphoid infection showing degeneration and desquamation of epithelial lining with denuded mucosa (arrow) and atrophy of intestinal glands. $H$ \& E x 400 . 
microbiota and morphology causing in vitro susceptibility for Salmonella Enteritidis attachment to the ileum in broilers.

\section{MacCallum goodpasture staining}

Intestinal sections which appeared as rod-shaped and pink in colour which confirms the presence of Gram-negative organisms in the intestine.

Hence, we can conclude from our study that even though the salmonellosis infection may not be the primary cause of death in birds, but it certainly contributes to the damage of the organs of alimentary system, therefore, affecting the overall health status of birds.

\section{CONCLUSION}

The present study revealed the presence of the paratyphoid bacteria in the poultry farms in Jabalpur region which may be gaining access to the human chain. Clinical signs along with characteristic microscopic lesions confirming paratyphoid infections in birds with gross gastrointestinal lesions were not observed were not considered to be of much significance for the appropriate diagnosis of paratyphoid infection, hence, isolation and identification by following gold standard procedure of culture and biochemical characterization becomes essential. The contaminated fecal samples from Salmonella in the poultry sheds and cages can further lead to the contamination of eggs and later chicks and hence control of this bacterial infection within the poultry sheds becomes a challenge.

\section{REFERENCES}

Aktar, N., Bilkis, R. and Ilias, M. (2016). Isolation and identification of Salmonella sp. from different food. International Journal of Biosciences. 8(2): 16-24.

Al Mamun, M.A., Kabir, S.L., Islam, M.M., Lubna, M., Islam, S.S., Akhter, A.T. and Hossain, M.M. (2017). Molecular identification and characterization of Salmonella species isolated from poultry value chains of Gazipur and Tangail districts of Bangladesh. African Journal of Microbiology Research. 11(11): 474-481.

Badhe, S.R., Fairoze, N. and Sudarshan, S. (2013). Prevalence of food borne pathogens in market samples of chicken meat in Bangalore, India. Indian Journal of Animal Research. 47(3): 262-264.

Burkholder, K.M., Thompson, K.L., Einstein, M.E., Applegate, T.J. and Patterson, J.A. (2008). Influence of stressors on normal intestinal microbiota, intestinal morphology and susceptibility to Salmonella Enteritidis colonization in broilers. Poultry Science. 87(9): 1734-1741.

Chakraborty, S., Roychoudhury, P., Samanta, I., Subudhi, P.K., Das, M., De, A. Bandyopadhayay, S., Joardar, S.N., Mandal, M., Qureshi, A. and Dutta, T.K. (2020). Molecular detection of biofilm, virulence and antimicrobial resistance associated genes of Salmonella serovars isolated from pig and chicken of Mizoram, India. Indian Journal of Animal Research. 54(5): 608-613.
Crhanova, M., Hradecka, H., Faldynova, M., Matulova, M., Havlickova, H., Sisak, F. and Rychlik, I. (2011). Immune response of chicken gut to natural colonization by gut microflora and to Salmonella enterica serovar Enteritidis infection. Infection and Immunity. 79(7): 2755-2763.

Deshmukh, S., Asrani, R.K., Ledoux, D.R., Rottinghaus, G.E., Bermudez, A.J. and Gupta, V.K. (2007). Pathologic changes in extrahepatic organs and agglutinin response to Salmonella Gallinarum infection in Japanese quail fed Fusarium verticillioides culture material containing known levels of fumonisin B1. Avian Diseases. 51(3): 705-712.

Dutta, P., Borah, M., Sarmah, R. and Gangil, R. (2013). Isolation of Salmonella Typhimurium from pigeons (Columba livia) in greater Guwahati, its histopathological impact and antibiogram. Comparative Clinical Pathology. 22: 147-150.

Dutta, P., Borah, M.K., Gangil, R. and Singathia, R. (2015). Gross/ histopathological impact of Salmonella Gallinarum isolated from layer chickens in Jaipur and their antibiogram assay. International Journal of Advanced Veterinary Science and Technology. 4(1): 153-159.

Faisal, S.M.W., Alam, A.K. and Sajed, M.N. (2017). Isolation and identification of Gram-negative bacteria from streetvended sauce and brand sauce in Dhaka city to evaluate their safety margin. The Pharma Innovation. 6(1): 68-72.

Garcia, K.O., Berchieri Jr, A., Santana, A.M., Alarcon, M.F.F., Freitas Neto, O.C. and Fagliari, J.J. (2013). Experimental infection of commercial layers with wild or attenuated Salmonella Gallinarum mutant strains: anatomic pathology, total blood cell count and serum protein levels. Brazilian Journal of Poultry Science. 15(2): 91-104.

Gast, R.K. (1997). Detecting infections of chickens with recent Salmonella Pullorum isolates using standard serological methods. Poultry Science. 76(1): 17-23.

Gast, R.K. (2013). Paratyphoid infections. In: Diseases of Poultry (13 ${ }^{\text {th }}$ Edn.). [Swayne, D.E. (ed.).] Wiley-Blackwell publishing, Ames, USA. pp. 693-713.

Gridley, M.F. (1960). Manual of Histological and Special Staining Technique, McGraw-hill Book Company., Inc. New York, Toronto, London.

Habib-ur-Rehman, S., Khan, H., Khan, S., Ahmad, N. and Bhatti, W.M. (2003). Incidence and gross pathology of salmonellosis in chicken in Hyderabad. Journal of Animal and Veterinary Advances. 2(10): 581-584.

Haider, M.G., Hossain, M.G., Hossain, M.S., Chowdhury, E.H., Das, P.M. and Hossain, M.M. (2004). Isolation and characterization of Enterobacteria associated with health and disease in Sonali chickens. Bangladesh Journal of Veterinary Medicine. 2(1): 15-21.

Havelaar, A.H., Kirk, M.D., Torgerson, P.R., Gibb, H.J., Hald, T., Lake, R.J., Praet, N., Bellinger, D.C., Silva, N.R., Gargouri, N. and Speybroeck, N. (2015). World Health Organization global estimates and regional comparisons of the burden of foodborne disease in 2010. PLoS Medicine. 12(12): e1001923.

Hossain, M.S., Chowdhury, E.H., Islam, M.M., Haider, M.G. and Hossain, M.M. (2006). Avian Salmonella infection: isolation and identification of organisms and histopathological study. Bangladesh Journal of Veterinary Medicine. 4(1): 7-12. 
Islam, M.M., Haider, M.G., Chowdhury, E.H., Kamruzzaman, M. and Hossain, M.M. (2006). Seroprevalence and pathological study of Salmonella infections in layer chickens and isolation and identification of causal agents. Bangladesh Journal of Veterinary Medicine. 4(2): 79-85.

ISO (2017). Microbiology of the food chain- horizontal method for the detection, enumeration and serotyping of Salmonellapart 1: detection of Salmonella sp.

Khasa, V., Singh, P., Mahajan, N.K. and Sonipat, H. (2018). Isolation and antibiotic sensitivity pattern of Salmonella enterica isolates from livestock and poultry of Haryana. International Journal of Health Sciences and Research. 8(12): 44-49.

Kumari, D., Mishra, S.K. and Lather, D. (2013). Pathomicrobial studies on Salmonella Gallinarum infection in broiler chickens. Veterinary World. 6(10): 725-729.

Long, M., Yu, H., Chen, L., Wu, G., Zhao, S., Deng, W., Chen, S., Zhou, K., Liu, S., He, L. and Ao, X. (2017). Recovery of Salmonella isolated from eggs and the commercial layer farms. Gut Pathogens. 9(1): 74.

Mansour, A.O., Al-Jbouri, S.A. and Mansour, H. (2013). Antimicrobial resistance pattern and RAPD profile of Salmonella Ohio isolated from broiler farms in Al-Najaf and Al-Muthana provinces. Kufa Journal for Veterinary Medical Sciences. 4(2): 18-30.

Mir, I.A., Kashyap, S.K. and Maherchandani, S. (2015). Isolation, serotype diversity and antibiogram of Salmonella enterica isolated from different species of poultry in India. Asian Pacific Journal of Tropical Biomedicine. 5(7): 561-567.

Muna, E.A., Salih, M.H., Zakia, A.M., Halima, M.O., Abeer, A.M., Ameera, M.M., Ali, H.O. and Idris, S.B. (2016). Pathology of broiler chicks naturally infected with Salmonella Enteritidis (S. Enteritidis) and Salmonella Typhimurium (S. Typhimurium) during an outbreak in Sudan. Journal of Scientific Research and Reports, 10(1): 1-8.

Ogunleye, A.O. and Carlson, S.A. (2012). Emergence of an SGI1bearing Salmonella enterica serotype Kentucky isolated from septic poultry in Nigeria. The Journal of Infection in Developing Countries. 6(6): 483-488.

OIE (2012). Fowl typhoid and pullorum disease Chapter 2.3. 11. In: OIE terrestrial Manual. pp. 1-14.
Prabakaran, R. (2014). Indian Poultry Industry: Current status, practical challenges and opportunities. In: World's Poultry Congress. September 2016. pp. 642-642.

Rahman, M.R., Shahinuzzaman, A.B.M., Saha, A.K., Sufian, M.A., Rahman, M.H. and Hossain, M.M. (2011). Prevalence of Salmonella infection in naturally infected layer of birds in Bangladesh. Bangladesh Veterinarian. 28(1): 8-18.

Rajagopal, R. and Mini, M. (2013). Outbreaks of salmonellosis in three different poultry farms of Kerala, India. Asian Pacific Journal of Tropical Biomedicine. 3(6): 496-500.

Ranjbar, V.R., Basiri, S. and Abbasi-Kali, R. (2020). Paratyphoid infection caused by Salmonella Typhimurium in a pigeon flock (Columbia livia) in Iran. Journal of Zoonotic Diseases. 4(1): 43-48.

Samanta, I., Joardar, S.N., Das, P.K., Sar, T.K., Bandyopadhyay, S., Dutta, T.K. and Sarkar, U. (2014). Prevalence and antibiotic resistance profiles of Salmonella serotypes isolated from backyard poultry flocks in West Bengal, India. Journal of Applied Poultry Research. 23(3): 536-545.

Sannat, C., Patyal, A., Rawat, N., Ghosh, R.C., Jolhe, D.K., Shende, R.K., Hirpurkar, S.D. and Shakya, S. (2017). Characterization of Salmonella Gallinarum from an outbreak in Raigarh, Chhattisgarh. Veterinary World. 10(2): 144.

Saravanan, S., Purushothaman, V., Murthy, T.R.G.K., Sukumar, K., Srinivasan, P., Gowthaman, V., Balusamy, M., Atterbury, R. and Kuchipudi, S.V. (2015). Molecular epidemiology of Non-typhoidal Salmonella in poultry and poultry products in India: implications for human health. Indian Journal of Microbiology. 55(3): 319-326.

Sharma, I. and Das, K. (2016). Detection of invA gene in isolated Salmonella from marketed poultry meat by PCR assay. Journal of Food Processing and Technology. 7(3): 1-4.

Snedecor, G.W. and Cochran, W.G. (1994). Statistical methods. Oxford and IBH Publishing Co., New Delhi.

Srinivasan, P., Balasubramaniam, G.A., Gopala, T.R., Murthy, K., Saravanan, S. and Balachandran, P. (2014). Prevalence and Pathology of salmonellosis in commercial layer chicken from Namakkal, India. Pakistan Veterinary Journal. 34(3): 324-328.

Tille, P. (2017). Bailey and Scott's diagnostic microbiology. (14 $4^{\text {th }}$ Edn.). Elsevier, St. Louis.pp. 75-78. 